\title{
Исследование однородности состава по толщине слоев GalnAsP, полученных на подложках InP методом газофазной эпитаксии
}

\author{
(C) Г.С. Гагис ${ }^{1}$, Р.В. Левин ${ }^{1}$, А.Е. Маричев ${ }^{1}$, Б.В. Пушный ${ }^{1}$, М.П. Щеглов ${ }^{1}$, Б.Я. Бер ${ }^{1}$, \\ Д.Ю. Казанцев ${ }^{1}$, Ю.А. Кудрявцев ${ }^{3}$, А.С. Власов ${ }^{1}$, Т.Б. Попова ${ }^{1}$, \\ Д.В. Чистяков ${ }^{4}$, В.И. Кучинский ${ }^{1,2}$, В.И. Васильев ${ }^{1}$ \\ 1 Физико-технический институт им. А.Ф. Иоффе Российской академии наук, \\ 194021 Санкт-Петербург, Россия \\ ${ }^{2}$ Санкт-Петербургский государственный электротехнический университет „ЛЭТИ“, \\ 197022 Санкт-Петербург, Россия \\ ${ }^{3}$ Cinvestav-IPN, \\ 2508 Cinvestav-IPN, Mexico \\ ${ }^{4}$ Университет ИТМО, \\ 197101 Санкт-Петербург, Россия \\ E-mail: galina.gagis@gmail.com
}

Поступила в Редакцию 17 июня 2019 г.

В окончательной редакции 25 июня 2019 г.

Принята к публикации 27 июня 2019 г.

Исследованы гетероструктуры GaInPAs/InP, полученные методом газофазной эпитаксии из металлорганических соединений при температуре $600^{\circ} \mathrm{C}$ и давлении 0.1 бар. Толщины выращенных слоев GaInAsP составляли $\sim 1$ мкм. Методом вторичной ионной масс-спектрометрии для твердых растворов $\mathrm{Ga}_{1-x} \operatorname{In}_{x} \mathrm{P}_{1-y} \mathrm{As}_{y}$ со средними составами $x=0.77-0.87, y=0.07-0.42$ выявлено изменение содержания атомов $\mathrm{V}$ группы $y$ по толщине эпитаксиального слоя на величину $\Delta y$ вплоть до 0.1 атомных долей в подрешетке элементов V группы. В большинстве случаев изменение у происходит в слое GaInAsP на протяжении до 200 нм от гетерограницы c InP. B отдельных случаях y изменяется на протяжении всего эпитаксиального слоя GaInPAs. Для эпитаксиальных слоев с удовлетворительным кристаллическим совершенством, величина $\Delta y$ была меньшей в случае лучшего согласования эпитаксиального слоя GaInPAs с подложкой. Для слоев GaInPAs, сильно рассогласованных с подложкой и характеризующихся низким кристаллическим совершенством, величина $\Delta y$ была близка к нулю. Все эти факты позволяют предположить, что на встраивание атомов V группы в формирующуюся кристаллическую решетку влияют упругие деформации, возникающие в формирующемся монослое, рассогласованном с ростовой поверхностью.

Ключевые слова: газофазная эпитаксия, твердые растворы, гетероструктуры, фотоэлектрические преобразователи.

DOI: 10.21883/FTP.2019.11.48446.9191

\section{1. Введение}

В связи с развитием технологий изготовления лазеров и фотоэлектрических преобразователей (ФЭП) открываются новые возможности для их применения, в частности, для передачи световой энергии по оптоволокну, либо через воздушное или космическое пространство. Например, для подзарядки беспилотных летательных аппаратов без необходимости их приземления может быть использован YAG: Nd-лазер с рабочей длиной волны 1064 нм [1]. Энергия фотона излучения данного лазера составляет 1.165 эВ, следовательно, в качестве активной области ФЭП необходим прямозонный материал с шириной запрещенной зоны 1.05-1.15 эВ. Согласно расчетам, приведенным в работе [2], такими материалами являются четверные твердые растворы $\mathrm{Al}_{u} \mathrm{In}_{1-u} \mathrm{As}_{w} \mathrm{Sb}_{1-w}$ $(0.3<u<0.5)$ и $\mathrm{Al}_{u} \operatorname{In}_{1-u} \mathrm{P}_{w} \mathrm{Sb}_{1-w}(0.2<u<0.4)$, изопериодичные с InAs и GaSb а также: $\mathrm{Al}_{u} \mathrm{Ga}_{1-u} \mathrm{As}_{w} \mathrm{Sb}_{1-w}$ $(0.2<u<0.3), \quad \mathrm{Al}_{u} \mathrm{Ga}_{w} \mathrm{In}_{1-u-w}$ As $\quad(0.2<u<0.3) \quad$ и $\mathrm{Ga}_{1-x} \operatorname{In}_{x} \mathrm{P}_{1-y} \mathrm{As}_{y}(x \approx 0.8, y=0.3-0.45)$, изопериодичные с InP. Однако в перечисленных системах, содержа- щих сурьму, согласно работе [3], имеются обширные области несмешиваемости, охватывающие интересующий нас диапазон составов. Кроме того, даже незначительное количество алюминия в процессе газофазной эпитаксии из металлорганических соединений (ГФЭМОС) способствует вхождению кислорода и углерода, что приводит к снижению подвижностей носителей заряда и возникновению фонового легирования. Поэтому предпочтение в настоящее время отдается безалюминиевым (Al-free) технологиям. С этой точки зрения подходящим материалом для ФЭП на длину волны 1064 нм остаются твердые растворы GaInAsP, изопериодичные с InP.

В настоящее время использование системы твердых растворов GaInAsP, получаемых на подложке InP, связано с изготовлением лазеров [4,5], где большей частью востребованы твердые растворы $\mathrm{Ga}_{1-x} \mathrm{In}_{x} \mathrm{P}_{1-y} \mathrm{As}_{y}$ c $y>0.46$ для активных областей, излучающих на длинах волн 1.2-1.55 мкм. Твердые растворы с составами $y<0.46$ используются для вспомогательных слоев, толщины которых не превышают 100 нм. В случае 
ФЭП требуются более толстые слои (более 500 нм) высокого кристаллического совершенства. Согласно работе [6], у полученных методом ГФЭМОС твердых растворов $\mathrm{Ga}_{1-x} \mathrm{In}_{x} \mathrm{P}_{1-y} \mathrm{As}_{y}$ при $y<0.44$ наблюдаются неоднородности в содержании компонентов V группы по площади структуры. Ранее мы обнаружили, что для таких твердых растворов с составами $x=0.77-0.87$, $y<0.45$ наблюдается так же неоднородность содержания элементов $\mathrm{V}$ группы по толщине эпитаксиального слоя $[7,8]$. Описанные в работе [6] условия выращивания близки к тем, что используются нами и другими исследователями [4], а именно давление $\sim 0.1$ бар, в качестве прекурсоров элементов V группы использованы гидриды $\mathrm{AsH}_{3}$ и $\mathrm{PH}_{3}$. В работе [6] приводится оценка степени неоднородности по площади эпитаксиальной структуры в зависимости от температуры роста и соотношения V/III. Так, согласно данным работы [6], для $\mathrm{V} / \mathrm{III}=80$ и температуры роста $600^{\circ} \mathrm{C}$ (что соответствует условиям получения большинства образцов из наших работ $[7,8])$ вариация содержания элементов $\mathrm{V}$ группы по площади эпитаксиальной структуры $\Delta y$ составит $\sim 0.02$ атомных долей в подрешетке элементов V группы.

В работах $[7,8]$, в которых представлены результаты исследований эпитаксиальных слоев $\mathrm{Ga}_{1-x} \mathrm{In}_{x} \mathrm{P}_{1-y} \mathrm{As}_{y}$ с составами $x=0.77-0.87,0.07<y<0.42$, методом вторичной ионной масс-спектрометрии (ВИМС) выявляется изменение содержания мышьяка $у$ по толщине эпитаксиального слоя на величину $\Delta y$ до 0.08 . Кроме того, в наших ранних исследованиях методом ВИМС выявлена неоднородность содержания элементов V группы по толщине эпитаксиальных слоев InAsPSb, полученных на InAs [9]. В работе [9] величины изменения атомных долей элементов $\mathrm{V}$ группы в подрешетке мы связывали с различием между равновесным параметром решетки эпитаксиального слоя $a_{f 0}$ и параметром решетки подложки $a_{s}$. За равновесный параметр решетки $a_{f 0}$ принимается параметр решетки твердого раствора, определяемый только его химическим составом, в отсутствие внешних воздействий в том числе в отсутствие деформаций, которые могут возникать в результате сопряжения с предыдущим слоем гетероструктуры в случае, если $a_{f 0}$ отличается от $a_{s}$. Для характеристики такого отличия принято использовать величину $(\Delta a / a)=\left[a_{s}-a_{f 0}\right] / a_{s}$. Параметр решетки $a_{s}$ в наших рассмотрениях мы считаем равным его равновесному значению, так как подложка имеет значительную толщину и поэтому мало подвержена воздействию растущей на ней эпитаксиальной пленки. Исследованные в данной работе материалы GaInAsP и InP имеют структуру цинковой обманки, равновесный параметр решетки таких веществ имеет одинаковые значения по всем направлениям семейства $\langle 001\rangle$. Однако, если эпитаксиальный слой сопряжен с подложкой, из-за несоответствия равновесных параметров решетки $(\Delta a / a)$ могут возникать деформации, в результате которых по каждому из направлений семейства $\langle 001\rangle$ параметр решетки будет иметь разную величину.
В данной работе мы продолжили исследовать эпитаксиальные слои $\mathrm{Ga}_{1-x} \mathrm{In}_{x} \mathrm{P}_{1-y} \mathrm{As}_{y}$, полученные на $\mathrm{InP}$, в том числе, в области составов с $y=0.94-0.95$.

\section{2. Методика эксперимента и анализа полученных результатов}

Эпитаксиальные слои $\mathrm{Ga}_{1-x} \mathrm{In}_{x} \mathrm{P}_{1-y} \mathrm{As}_{y}$ выращивались в широком диапазоне составов $x=0.53-0.87$, $y=0.07-0.95$ на подложках $\operatorname{InP}(001)$, легированных $\mathrm{Sn}$ до уровня $n=(1-3) \cdot 10^{18} \mathrm{~cm}^{-3}$, разориентированных на $4-4.5^{\circ}$ в направлении (111). Толщины подложек составляли 380-390 мкм. Эпитаксиальный рост осуществлялся на установке „AIX200“ фирмы AIXTRON при давлении 0.1 бар и температуре $600^{\circ} \mathrm{C}$. Температура выбиралась с таким расчетом, чтобы в дальнейшем выбранные в результате наших экспериментов эпитаксиальные режимы можно было использовать для выращивания структур, содержащих слои, легированные цинком. Известно, что цинк склонен к интенсивной диффузии. Снижение температуры эпитаксиального роста позволяет снизить диффузионные процессы, однако температура все же должна быть достаточной для обеспечения требуемых химических реакций в процессе разложения прекурсоров и формирования эпитаксиальной пленки.

В качестве газа-носителя применялся водород с точкой росы не выше $-100^{\circ} \mathrm{C}$, общий поток водорода через реактор составлял 5 л/мин. В качестве прекурсоров атомов III группы использовались триэтилгаллий TEGa и триметилиндий TMIn, находящиеся при температуре $17^{\circ} \mathrm{C}$, давление в барботере с TEGa coставляло 1 бар, с TMIn - 0.3 бар. В качестве прекурсоров атомов V группы использовались арсин $\mathrm{AsH}_{3}$ и фосфин $\mathrm{PH}_{3}$. В процессе нагрева реактора, после достижения температуры выше $400^{\circ} \mathrm{C}$, в реактор подавалось 268 мкмоль/мин фосфина с целью предотвращения потери фосфора поверхностью подложки InP. Перед получением эпитаксиальных слоев GaInPAs, выращивались буферные слои InP в течение 10-20 мин. При формировании буферного слоя поток фосфина составлял $X_{\mathrm{PH} 3}=7800$ мкмоль/мин, триметилиндия $X_{\mathrm{TMIn}}=26$ мкмоль/мин $(\mathrm{V} / \mathrm{III}=300)$. После получения буферного слоя через реактор в течение одной минуты продувался чистый водород для полной замены газовой смеси, после чего осуществлялось выращивание эпитаксиального слоя GaInPAs. Было получено три серии образцов: А, В, C.

При выращивании образцов серии А, потоки прекурсоров составляли $X_{\mathrm{TEGa}}=7$ мКмоль $/$ мин, $X_{\mathrm{TMIn}}=$ $=30-32$ мкмоль $/$ мин, $X_{\mathrm{PH} 3}=3350$ мкмоль $/$ мин, $X_{\mathrm{AsH} 3}=$ $=9-31$ мкмоль/мин, скорости роста при этом были около 20 нм/мин.

Для серии В потоки прекурсоров лежали в диапазоне: $X_{\mathrm{TEGa}}=2-3$ мкмоль/мин, $\quad X_{\mathrm{TMIn}}=14-18$ мкмоль $/$ мин, $X_{\mathrm{AsH} 3}=16-31$ мкмоль/мин, $X_{\mathrm{PH} 3}=848-1696$ мкмоль/мин. Скорости роста составили $10-14$ нм/мин. 
Серия С отвечает области составов $y=0.92-0.95$, образец С1 был получен при $X_{\mathrm{TEGa}}=2$ мкмоль/мин, $X_{\mathrm{TMIn}}=11.5$ мкмоль/мин, $X_{\mathrm{PH}_{3}}=1696$ мкмоль/мин, $X_{\mathrm{AsH}_{3}}=$ $=90$ мкмоль/мин, образцы С2 и С3 выращивались при $X_{\mathrm{TEGa}}=11-13$ мкмоль $/$ мин,$\quad X_{\mathrm{TMIn}}=17.6$ мкмоль $/$ мин , $X_{\mathrm{PH} 3}=714$ мкмоль/мин, $X_{\mathrm{AsH} 3}=90$ мкмоль/мин. Скорости роста составили $15-20$ нм/мин.

Эпитаксиальные слои GaInPAs у образцов серии A выращивались в течение 45 мин, серий В и С -60 мин. В течение времени осаждения эпитаксиального слоя GaInPAs в реакторе поддерживалась постоянная температура и неизменные значения потоков прекурсоров и водорода.

Толщины полученных эпитаксиальных слоев лежали в пределах 0.6-1.2 мкм.

Составы эпитаксиальных слоев GaInPAs определялись методом рентгеновского микроанализа на установке „Camebax“ фирмы Cameca c ускоряющим потенциалом $10 \mathrm{\kappa B}$, что соответствует усреднению состава на глубине 500 нм. Погрешность измерений состава мы оцениваем как \pm 0.015 атомных долей в подрешетке.

Степень кристаллического совершенства полученных образцов оценивалась по результатам исследования фотолюминесценции (ФЛ) и рентгеновской дифрактометрии ( $X$-ray diffractometry - XRD), однородность распределения компонентов In, Ga, P и As по толщине эпитаксиального слоя была исследована методом вторичной ионной масс-спектрометрии (ВИМС).

Спектры ФЛ возбуждались с помощью YAG : Nd-лазера $(\lambda=532 \mathrm{Hм})$ и регистрировались фотоприемником на основе $\mathrm{PbS}$ для диапазона $1.2-2$ мкм и ФЭУ-62 для диапазона 0.58-1.2 мкм при температуре жидкого азота $77 \mathrm{~K}$.

Исследование структуры эпитаксиальных слоев методом XRD проводилось на базе трехкристального рентгеновского дифрактометра, ДРОН-2“ с использованием симметричных рефлексов типа (004) в $\mathrm{Cu} K_{\alpha 1}$-излучении, измерялись интенсивности отражения от плоскостей (001) для различных углов падения рентгеновского луча $\theta$. Кривые рентгеновского дифракционного отражения (XRD-кривые) снимались в режиме $\theta / 2 \theta$. Кристаллическое совершенство и однородность эпитаксиальных слоев характеризуются полными ширинами на половине высоты максимума (Full Width at Half Maximum FWHM) пиков отражений от подложки $\alpha_{s}$ и от эпитаксиального слоя GaInPAs $\alpha_{f}$. При оценке качества полученных образцов, мы ориентируемся на минимальные измеренные в настоящей работе значения FWHM: $\alpha_{s}=15-30^{\prime \prime}, \alpha_{f}<50^{\prime \prime}$. Большие значения $\alpha_{f}$ при достаточно высоких интенсивностях отражений, скорее всего, связаны с неоднородностью состава эпитаксиального слоя и, как следствие, наличием у него целого спектра значений параметра решетки $a_{f}$. При трехкристальной схеме регистрации XRD-кривых каждому углу отражения $\theta$ можно сопоставить величину относительного несоответствия параметров решетки эпитаксиального слоя GaInAsP и подложки $(\Delta a / a)_{\perp}=\left[a_{f \perp}-a_{s}\right] / a_{s}$ по

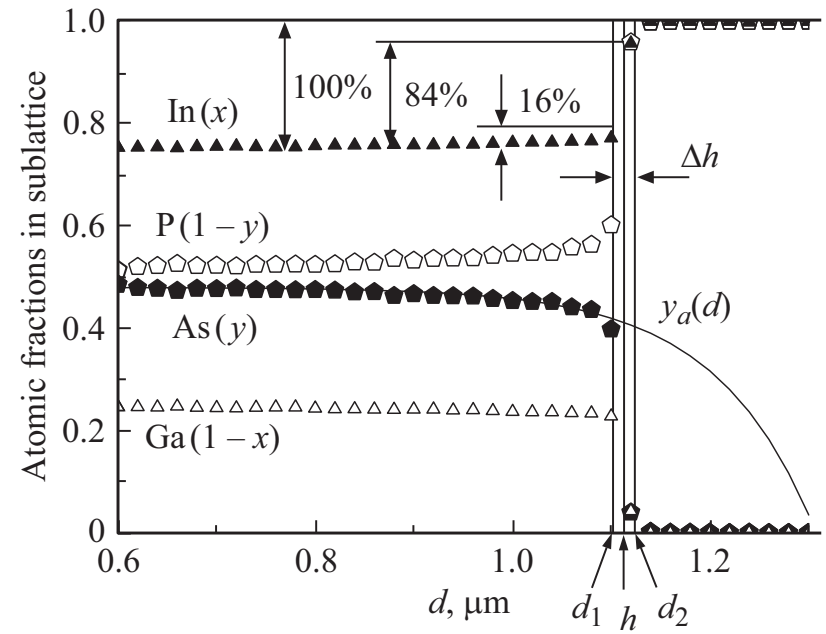

Рис. 1. Фрагмент профиля ВИМС, типичного для исследованных в настоящей работе гетероструктур GaInAsP/InP (на примере образца А2). $d$ - глубина, отсчитываемая от поверхности эпитаксиального слоя.

направлению [001], где $a_{f \perp}-$ величина параметра решетки по направлению [001] для некой бесконечномалой области внутри эпитаксиального слоя, $a_{s}$ - параметр решетки подложки InP.

Если слой однороден, то все области в нем имеют один и тот же параметр решетки, который можно сопоставить положению максимума пика интенсивности отражения от слоя, сам пик при этом будет узким. В случаях, когда пики отражения от слоя сильно уширены и имеют несимметричный характер, в качестве характеристики эпитаксиальных слоев мы используем усредненную величину $(\Delta a / a)_{\perp}^{\mathrm{M}}$, определяемую по центру тяжести пика отражения от слоя.

Пики отражений от слоя для структур низкого кристаллического совершенства характеризуются низкой интенсивностью и, как правило, не различимы с использованием трехкристальной схемы, а двухкристальная схема не позволяет однозначно связать $\theta$ и $(\Delta a / a)_{\perp}$, но может быть использована для оценки величины $(\Delta a / a)_{\perp}^{\mathrm{M}}$.

Измерения ВИМС осуществлялись на базе установки „Cameca IMS 7f“. На рис. 1 показан профиль ВИМС, типичный для исследованных в данной работе образцов. Согласно данным ВИМС, содержание элементов III группы оставалось постоянным по толщине всего эпитаксиального слоя GaInPAs (зафиксированные в отдельных случаях изменения не превышали 0.01 атомных долей в подрешетке III группы, и ими мы в данных рассмотрениях пренебрегаем), поэтому профиль содержания элементов третьей группы был использован для определения условного положения гетерограницы по критерию 16-84\%, при котором средние величины содержания индия в слое твердого раствора GaInAsP и в слое InP берутся за номинальные величины. Далее определяются координаты по глубине исследуемого 
образца $d_{1}$ и $d_{2}$, на которых изменение содержания индия составит 16 и 84\% от общей разницы между номинальными значениями. Условное положение гетерограницы определяется при этом как $h=\left(d_{1}+d_{2}\right) / 2$. Если начало отсчета $d=0$ соответствует поверхности исследуемого образца, то $h$ будет соответствовать условной толщине эпитаксиального слоя. Качество эпитаксиальных образцов характеризуется также величиной размытия гетерограницы $\Delta h=d_{2}-d_{1}$ (рис. 1), которая зависит от кристаллического совершенства эпитаксиальной структуры. Для лучших исследованных в данной работе образцов $\Delta h$ составило 15-20нм.

Содержание элементов V группы можно охарактеризовать атомными долями мышьяка в подрешетке элементов V группы $y$. Проанализировав все полученные в данной работе профили ВИМС, мы пришли к выводу, что полученные методом ВИМС зависимости атомной доли мышька в подрешетке $\mathrm{V}$ группы $y$ от глубины эпитаксиального слоя $d$ могут быть аппроксимированы выражениями вида

$$
y_{a}(d)=y_{\infty}+\Delta y_{e} \exp \left[(d-h) / h_{d}\right]+k d,
$$

где $y_{\infty}-$ асимптотическое значение для экспоненциальной составляющей (точность определения \pm 0.015 ), $\Delta y_{e}$ - масштабный множитель (точность \pm 0.005 ), $h_{d}$ - характеристическое расстояние (точность $\pm 5 \mathrm{HM}$ при $h_{d}>3 \Delta h$, иначе считаем $\Delta y_{e}=0$ и $\left.h_{d}=0\right), k-$ коэффициент наклона (точность $\pm 5 \cdot 10^{-6} \mathrm{HM}^{-1}$ ). В скобках указаны точности определения коэффициентов по среднеквадратичному отклонению полученных экспериментальных данных от зависимости (1). Типичное значение $h_{d}$ составило 50-100 нм, максимальное $-200 \mathrm{Hм.}$ Таким образом, поскольку $h_{d} \ll h$, можно считать что $y_{\infty}$ - это содержание мышьяка у поверхности эпитаксиального слоя GaInAsP.

В качестве общей характеристики изменения содержания элементов V группы по толщине всего слоя GaInAsP введем величину: $\Delta y=\Delta y_{e}+k h$. У всех рассмотренных в данной работе образцов коэффициенты $\Delta y_{e}$ имеют отрицательное значение. Величина $k$ в большинстве случаев отрицательна, но для некоторых образцов принимала и положительные значения.

\section{3. Анализ полученных результатов}

На рис. 2 изображен концентрационный квадрат твердых растворов $\mathrm{Ga}_{1-x} \operatorname{In}_{x} \mathrm{As}_{y} \mathrm{P}_{1-y}$. Линии изопериода с $\mathrm{InP}$ и $\mathrm{GaAs}\left(\mathrm{LM}_{\mathrm{GaAs}}\right.$ и $\left.\mathrm{LM}_{\mathrm{InP}}\right)$, изоэнергетические линии для прямого энергетического зазора Г и область непрямозонных составов (I) рассчитаны с использованием данных работы [10]. В концентрационном квадрате на рис. 2 также показаны измеренные на „Сamebax“ составы исследованных в данной работе образцов.

Образцы, для которых выполнялось условие $-4 \cdot 10^{-3}<(\Delta a / a)_{\perp}^{\mathrm{M}}<2.5 \cdot 10^{-3}$, имели удовлетворительное кристаллическое совершенство, которое

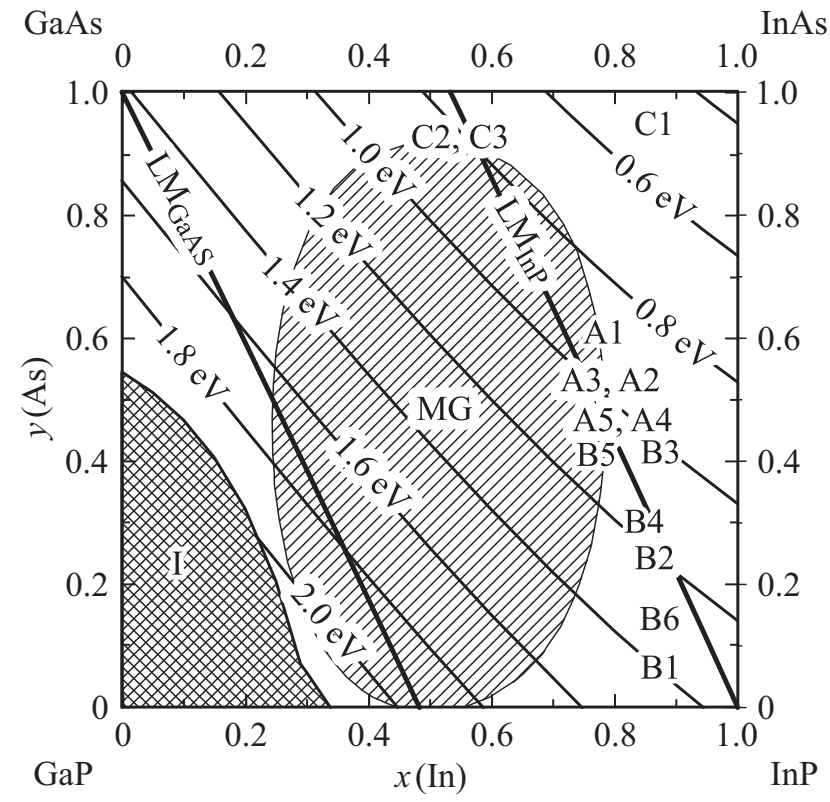

Рис. 2. Концентрационный квадрат твердых растворов $\mathrm{Ga}_{1-x} \operatorname{In}_{x} \mathrm{As}_{y} \mathrm{P}_{1-y}, \mathrm{MG}$ - область спинодального распада при $600^{\circ} \mathrm{C}[3]$.

характеризуется достаточно интенсивными пиками отражения от слоя на XRD-кривых. FWHM пиков отражения от слоя $\alpha_{f}$ при этом могли достигать до 200" в случаях, когда методом ВИМС выявлялась существенная (c $\Delta y>0.05)$ неоднородность содержания элементов V группы $y$, характеризующаяся значениями коэффициента наклона $k=-(7-8) \cdot 10^{-5} \mathrm{Hм}^{-1}$. В таких случаях кристаллическое совершенство полученных образцов мы считали удовлетворительным, если размытие гетерограницы $\Delta h$ не превышало 20 нм, и вместе с этим при $77 \mathrm{~K}$ наблюдалась ФЛ, которую можно связать с межзонными переходами. При типичных для исследованных образцов $k=-(1-2) \cdot 10^{-5} \mathrm{HM}^{-1}, h_{d}=70-200 \mathrm{Hм}$, $\Delta y_{e}=0.03-0.09$ неоднородность содержания элементов $\mathrm{V}$ группы можно считать существенной для отдельной области эпитаксиального слоя GaInPAs, прилегающей к гетерогранице с InP, а основную часть слоя GaInPAs можно считать относительно однородной.

На рис. 3 приводятся XRD-кривые (рис. 3, $a$ ) и спектры ФЛ (рис. 3,b) для двух близких по составу образцов А2 и А3. У образца А2 методом ВИМС выявлена достаточно существенная неоднородность $у$ по толщине слоя GaInPAs (см. рис. 1): $\Delta y=-0.09$, а у образца А3 профиль ВИМС по толщине слоя GaInPAs был практически однородным. Как можно видеть, неоднородность по толщине эпитаксиального слоя приводит к уширению пиков отражения от слоя GaInAsP на кривых XRD и к уширению пиков спектров ФЛ.

Для образцов серий А и В (средние составы $y<0.55)$ прослеживалась связь между степенью и характером неоднородности и величиной $\left|(\Delta a / a)_{\perp}^{\mathrm{M}}\right|$. Образцы с $\left|(\Delta a / a)_{\perp}^{\mathrm{M}}\right| \geq 9 \cdot 10^{-3}$ характеризовались достаточно раз- 


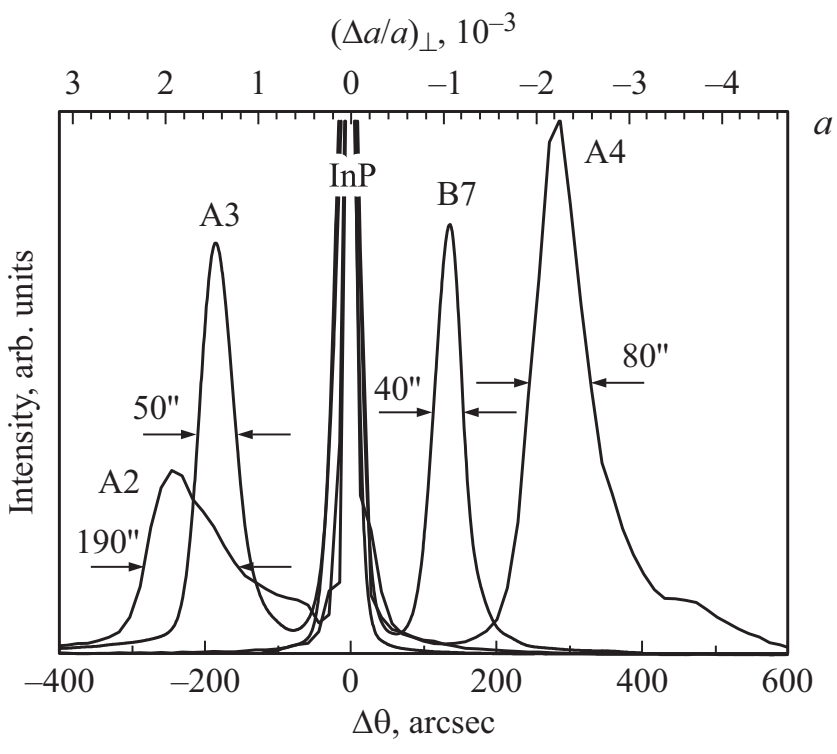

Wavelength, $\mu \mathrm{m}$

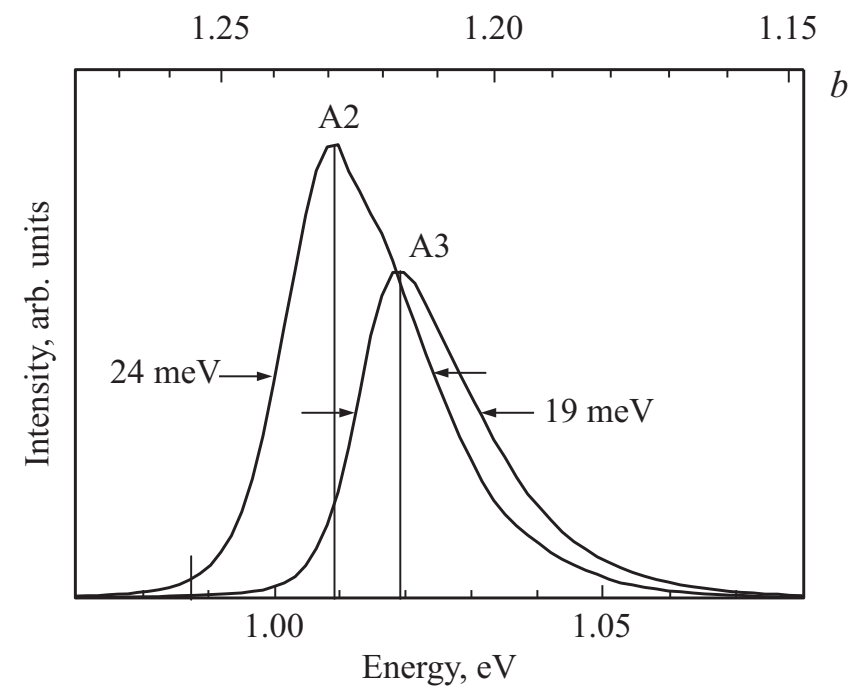

Рис. 3. Кривые XRD для образцов $\mathrm{A} 3 \quad(\Delta y \approx 0), \mathrm{A} 2$ $(\Delta y=-0.08)$, А4 $(\Delta y=-0.06)$ и образца В7 $(a)$, а также спектры ФЛ для образцов А3 и А2 $(b)$.

мытыми гетерограрицами: $\Delta h=30$ нм. Для образца В6 c $(\Delta a / a)_{\perp}^{\mathrm{M}}=-1.2 \cdot 10^{-2}$ наблюдалась максимальная величина $\Delta y=0.11$, причем изменение происходило на протяжении всего эпитаксиального слоя. Для образцов c $\left|(\Delta a / a)_{\perp}^{\mathrm{M}}\right|>1.2 \cdot 10^{-2}$ (например, для образца В1 $\left.(\Delta a / a)_{\perp}^{\mathrm{M}}=-1.3 \cdot 10^{-2}\right)$ основное изменение содержания элементов V группы происходило на участке размытия гетерограницы, а по толщине эпитаксиального слоя величина у оставалась практически постоянной.

Исследования методом XRD для образцов с низкой степенью кристаллического совершенства проводились при использовании двухкристальной схемы, и величина $(\Delta a / a)_{\perp}^{\mathrm{M}}$ для них не может быть оценена с достаточной точностью, а наблюдаемые высокие значения $\alpha_{f} \approx 1000^{\prime \prime}$ не могут быть однозначно связаны с на- личием градиента по параметру решетки. В случаях, когда ВИМС не выявляет неоднородностей состава по толщине слоя GaInAsP у образца с низкой степенью кристаллического совершенства, исследованного с использованием двухкристальной схемы, большие величины $\alpha_{f}$, скорее всего. связаны с большим количеством кристаллических дефектов.

Большие характеристические расстояния $h_{d}=$ $=100-200 \mathrm{нм}$ и $\Delta y_{e}=-0.05--0.04$ наблюдались у образцов серии А с $-4 \cdot 10^{-3}<(\Delta a / a)_{\perp}^{\mathrm{M}}<0$. Большая величина наклона $|k|=(3-8) \cdot 10^{-5} \mathrm{Hм}^{-1}$ проявилась у образцов серии $\mathrm{B}$, для которых $(\Delta a / a)_{\perp}^{\mathrm{M}} \leq-4 \cdot 10^{-3}$, причем наклон был как положительным, так и отрицательным. В случае отрицательного наклона и ощутимого вклада экспоненциальной составляющей выражения (1) на протяжении всего эпитаксиального слоя $\mathrm{Ga}_{1-x} \mathrm{In}_{x} \mathrm{As}_{y} \mathrm{P}_{1-y}$ происходит довольно ощутимое изменение величины $y$, как это было у образцов В3 (рис. $4, a)$ и В6. Однако возможны случаи с положительным наклоном, как это имело место для образцов В4 (рис. $4, b)$ и В5, в таких случаях $\Delta y=-0.01--0.02$.

Для образца В7 с высокой степенью согласования слоев GaInAsP и InP $(\Delta a / a)_{\perp}^{\mathrm{M}}=-1 \cdot 10^{-3}$, на XRD-кривой наблюдается узкий и симметричный пик (рис. 3,a), свидетельствующий о высокой степени однородности и высоком кристаллическом совершенстве эпитаксиального слоя.

В серии А даже у образцов с $-2.5 \cdot 10^{-3}<$ $<(\Delta a / a)_{\perp}^{\mathrm{M}}<0 \quad$ (образцы $\left.\mathrm{A} 4, \mathrm{~A} 5\right)$ и $\alpha_{f}<80^{\prime \prime}$, на $\mathrm{XRD}$-кривых пики отражения от слоя имели плато со стороны отрицательных значений $(\Delta a / a)_{\perp}$, что можно видеть для образца А4 (рис. 3,a). Подобное плато наблюдалось и у образца А5, хотя для него $(\Delta a / a)_{\perp}^{\mathrm{M}}=0$. Согласно исследованиям ВИМС, оба образца имели достаточно высокую неоднородность содержания мышьяка по толщине $\Delta y=0.05-0.06$ на достаточно протяженных расстояниях $\left(h_{d}=100-200 \mathrm{HM}\right)$. Известно, что гетероструктуры $\mathrm{Ga}_{1-x} \operatorname{In}_{x} \mathrm{As}_{y} \mathrm{P}_{1-y} / \operatorname{InP}(100)$, выращенные при температурах $580-650^{\circ} \mathrm{C}$, имеют хорошее качество, если их $(\Delta a / a)_{\perp}$ при комнатной температуре попадает в интервал от $-4 \cdot 10^{-3}$ до $+2 \cdot 10^{-3}[11,12]$. При температуре эпитаксии этот интервал, вероятно, симметричен относительно нуля, а при понижении температуры сдвигается в сторону отрицательных значений из-за различия в коэффициентах термического расширения GaInAsP и InP. Неоднородность у образца А5 можно связать с тем, что при температуре эпитаксии рассогласование для него было более существенным, нежели при комнатной температуре, в то время как для более однородного образца В7 при температуре эпитаксии рассогласование могло быть оптимальным. Также различие в формах

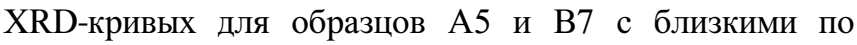
значению величинами $(\Delta a / a)_{\perp}^{\mathrm{M}}$ может быть обусловлено режимами роста: у образцов серии А скорости роста были в $\sim 2$ раза выше скоростей роста образцов серии В.

Для образцов серии С неоднородностей содержания элементов V группы по толщине не выявлено. Для 
образца С1 величина $(\Delta a / a)$, рассчитанная по составу, достаточно велика и составляет $2 \cdot 10^{-2} \pm 3 \cdot 10^{-3}$. Согласно данным ВИМС, размытие гетерограницы для образца $\mathrm{C} 1$ составляет $\Delta h=120$ нм, однако в данном случае столь большую величину $\Delta h$ можно связать с весьма ощутимым рельефом поверхности, который исследовался при помощи сканирующего электронного микроскопа (СЭМ), при этом на сколе прослеживались кратеры глубиной до 200 нм.

Следует отметить, что для всех исследованных в настоящей работе образцов в случае наличия неоднородности содержания элементов V группы по толщине слоя наблюдалось повышенное содержание фосфора возле гетерограницы c InP и более низкое его содержание в глубине слоя. В работе [9], в которой исследовались твердые растворы InAsPSb, выращенные на подложках InAs, для неоднородных по составу слоев InAsPSb возле гетерограницы наблюдалось повышенное содержание мышьяка. Возможно, это связано с взаимодействием элементов V группы газовой фазы с одноименными элементами в кристаллической решетке. В работе [13] отмечается, что при димеризации элементов V группы на ростовой поверхности химические связи возникают именно между одноименными элементами.

На рис. 4 для представленных образцов показаны рассчитанные по составу величины рассогласования слоя и подложки $f(d)=\left[a_{f}\left(x, y_{a}(d)\right)-a_{\mathrm{InP}}\right] / a_{\mathrm{InP}}+\Delta f$, где $x$ - измеренное методом рентгеновского микроанализа содержание индия в эпитаксиальном слое $\mathrm{Ga}_{1-x} \mathrm{In}_{x} \mathrm{As}_{y} \mathrm{P}_{1-y}, y_{a}(d)$ - аппроксимация содержания мышьяка по выражению (1), $\Delta f-$ поправка, которую необходимо внести в расчеты для того, чтобы устранить систематическую погрешность, связанную с точностью и особенностями измерений методом рентгеновского микроанализа. При внесении поправки $\Delta f$ мы ориентировались на данные рентгеновской дифрактометрии, считая, что основной объем эпитаксиального слоя не деформирован, и согласно этому допущению полагали, что измеренная величина $(\Delta a / a)_{\perp}^{\mathrm{M}}$ отвечает среднему равновесному параметру решетки эпитаксиального слоя.

Как можно видеть на рис. 4, $a$, для образца В3 возле гетерограницы величина рассогласования $|f(d)|$ меньше, чем на удалении от нее, а для В4 (рис. $4, b)$ - наоборот. Возможно, такие результаты расчета для образца В4 связаны с тем, что на гетерогранице с InP формируется деформированный эпитаксиальный слой, растянутый по плоскости (001), и такая деформация обеспечивает лучшее сопряжение кристаллической решетки твердого раствора GaInPAs с кристаллической решеткой InP. В работе [13] отмечается, что подобные деформации влияют на встраивание элементов V группы в формирующуюся кристаллическую решетку. По мере осаждения слоя за счет возрастающих упругих напряжений должны возникать вакансии несоответствия, частично снимающие упругие деформации, что в свою очередь должно сказываться на характере встраивания компонентов $\mathrm{V}$ группы в формирующуюся кристаллическую решетку,
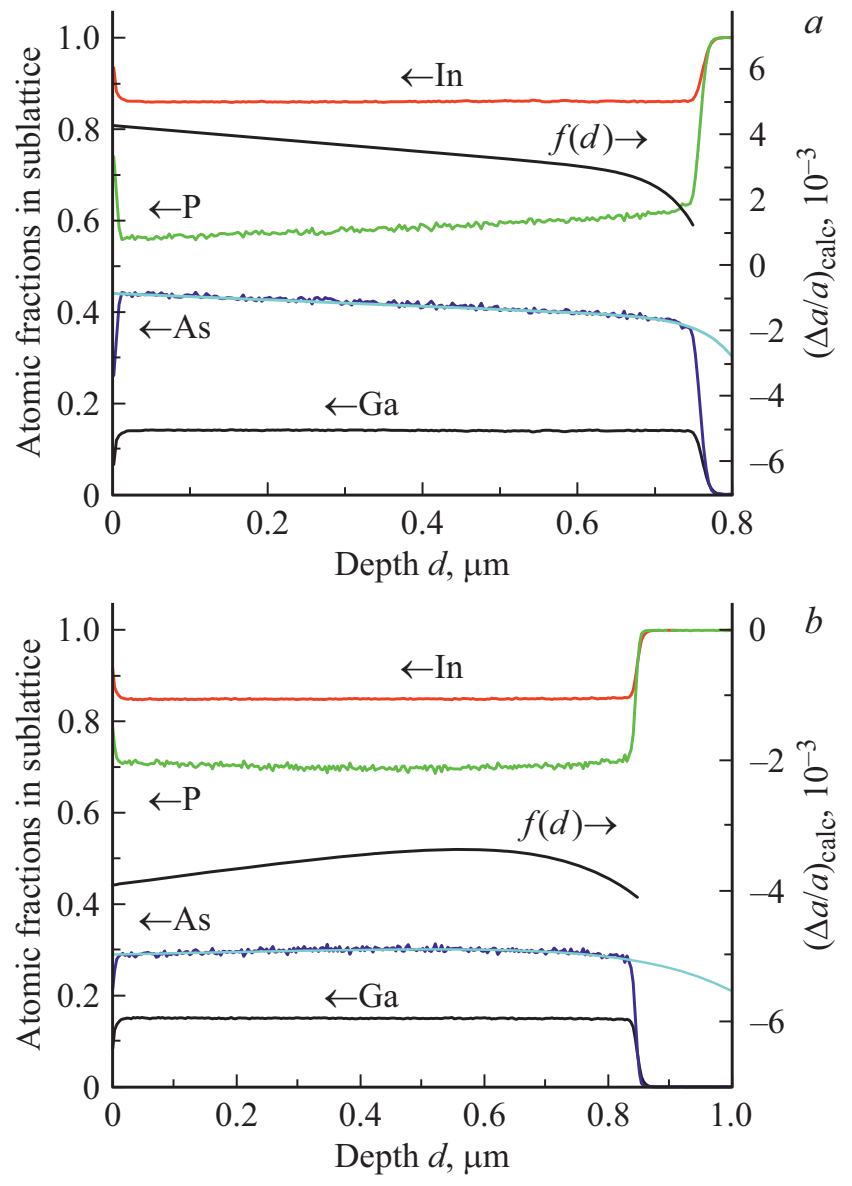

Рис. 4. Профили ВИМС для образца В3 с $k=-7 \cdot 10^{-5} \mathrm{HM}^{-1}(a)$ и В4 с $k=+3 \cdot 10^{-5} \mathrm{HM}^{-1}(b)$.

поэтому по ходу осаждения эпитаксиального слоя состав подрешетки V группы меняется.

\section{4. Заключение}

В результате проведенных исследований выявлено, что неоднородности в эпитаксиальных слоях GaInAsP возникают в случае, когда состав газовой смеси не соответствует составу твердого раствора, идеально согласованного с подложкой при температуре роста. При этом величина $\left|(\Delta a / a)_{\perp}^{\mathrm{M}}\right|$ должна попадать в определенный интервал при температуре эпитаксиального роста. Если обобщить полученные нами данные, то такой интервал можно оценить как $\left|(\Delta a / a)_{\perp}^{\mathrm{M}}\right|<1 \cdot 10^{-2}$ при температуре эпитаксии. При комнатной температуре он будет сдвинут в сторону отрицательных значений на величину $\sim 1 \cdot 10^{-3}$. Определенный нами интервал рассогласований слоя и подложки $-4 \cdot 10^{-3}<(\Delta a / a)_{\perp}^{\mathrm{M}}<2.5 \cdot 10^{-3}$, в котором полученные образцы серий А и В имеют удовлетворительную степень кристаллического совершенства, соответствует данным работ $[11,12]$. Если анализировать свойства эпитаксиальных слоев GaInPAs, близких по составу и выра- 
щенных при схожих условиях, можно проследить связь между величиной $(\Delta a / a)_{\perp}^{\mathrm{M}}$ и однородностью содержания элементов V группы по толщине эпитаксиального слоя, что позволяет сделать вывод о влиянии возникающих в процессе роста деформаций кристаллической решетки на встраивание элементов V группы.

\section{Благодарности}

Авторы работы выражают глубокую благодарность своему коллеге из ФТИ им. А.Ф. Иоффе С.И. Трошкову за сделанные при помощи СЭМ снимки сколов и поверхностей образцов.

\section{Финансирование работы}

Исследования ВИМС, СЭМ и „Саmebax“ проводились с использованием оборудования ЦКП „Материаловедение и диагностика в передовых технологиях“ (ФТИ им. А.Ф. Иоффе), поддерживаемом Министерством образования и науки РФ (уникальный идентификатор проекта RFMEFI62117X0018).

\section{Конфликт интересов}

Авторы заявляют об отсутствии конфликта интересов.

\section{Список литературы}

[1] Y. Akiyama, M. Sasaki, H. Yuasa, N. Nishida. LaserOpto, 33 (4), 46 (2001).

[2] A. Sasaki. Jpn. J. Appl. Phys., 19 (9), 1695 (1979).

[3] K. Onabe. Jpn. J. Appl. Phys., 21 (6), L323 (1982).

[4] Po-Hsun Lei. Optics Commun., 273, 532 (2007).

[5] M. Razeghi. MOCVD Challenge. Survey of GaInPInP\&GaInAsP-GaAs for photonic and electronic device applications. 2edn. (Taylor and Francis, CRC Press, 2010) p. 773.

[6] W.L. Holstein. J. Cryst. Growth, 167, 525 (1996).

[7] В.И. Васильев, Г.С. Гагис, Р.В. Левин, А.Е. Маричев, Б.В. Пушный, М.П. Щеглов, В.И. Кучинский, Б.Я. Бер, Д.Ю. Казанцев, А.Н. Горохов, Т.Б. Попова. Письма ЖТФ, 44 (24), 17 (2018). [V.I. Vasil'ev, G.S. Gagis, R.V. Levin, A.E. Marichev, B.V. Pushnyi, M.P. Scheglov, V.I. Kuchinskii, B.Ya. Ber, D.Yu. Kazantsev, A.N. Gorokhov, T.B. Popova. Techn. Phys. Lett., 44 (12), 1127 (2018)].

[8] A.E. Marichev, R.V. Levin, B.V. Pushnyii, G.S. Gagis, V.I. Vasil'ev, M.P. Scheglov, D.Yu. Kazantsev, B.Ya. Ber, T.B. Popova, E.P. Marukhina. J. Phys.: Conf. Ser., 1135, 012076 (2018).

[9] В.И. Васильев, Г.С. Гагис, Р.В. Левин, В.И. Кучинский, А.Г. Дерягин, Д.Ю. Казанцев, Б.Я. Бер. Письма ЖТФ, 43 (19), 78 (2017). [V.I. Vasil'ev, G.S. Gagis, R.V. Levin, V.I. Kuchinskii, A.G. Deryagin, D.Yu. Kazantsev, B.Ya. Ber. Tech. Phys. Lett., 43 (10), 905 (2017)].

[10] I. Vurgaftman, J.R. Meyer, L.R. Ram-Mohan. J. App. Phys., 89, 5815 (2001).

[11] K. Nakajima, S. Yamazaki, S. Komiya, K. Akita. J. Appl. Phys., 52, 4575 (1981).
[12] A. Mircea, R. Mellet, B. Rose, D. Robein, H. Thibierge, G. Leroux, P. Daste, S. Godefroy, P. Ossart, A.M. Pougnet. J. Electron. Mater., 15 (4), 205 (1986).

[13] J.E. Cunningham, M.B. Santos, K.W. Goossen, M.D. Williams, W. Jan. Appl. Phys. Lett., 64, 2418 (1994).

Редактор А.Н. Смирнов

\section{Investigation of composition uniformity on thickness of GalnAsP layers grown on InP substrates by vapor-phas epitaxy}

\author{
G.S. Gagis ${ }^{1}$, R.V. Levin ${ }^{1}$, A.E. Marichev' ${ }^{1}$, \\ B.V. Pushnyii ${ }^{1}$, M.P. Scheglov ${ }^{1}$, B.Ya. Ber ${ }^{1}$, \\ D.Yu. Kazantsev ${ }^{1}$, Yu.A. Kudriavtsev ${ }^{3}$, \\ A.S. Vlasov' ${ }^{1}$, T.B. Popova ${ }^{1}$, D.V. Chistyakov ${ }^{4}$, \\ V.I. Kuchinskii ${ }^{1,2}$, V.I. Vasil'ev' \\ ${ }^{1}$ loffe Institute, \\ 194021 St. Petersburg, Russia \\ 2 St. Petersburg Electrotechnical University „LETI“, \\ 197022 St. Petersburg, Russia \\ ${ }^{3}$ Cinvestav-IPN, \\ 2508 Cinvestav-IPN, Mexico \\ ${ }^{4}$ ITMO University, \\ 197101 St. Petersburg, Russia
}

Abstract GaInPAs/InP heterostructures grown by low pressure $\left(0.1 \mathrm{bar}, 600^{\circ} \mathrm{C}\right)$ metal-organic chemical vapor phase deposition were investigated. The thicknesses of grown GaInAsP layers were about $1 \mu \mathrm{m}$. For the epitaxial layers $\mathrm{Ga}_{1-x} \operatorname{In}_{x} \mathrm{P}_{1-y} \mathrm{As}_{y}$ with average compositions of $x=0.77-0.87$ and $y=0.07-0.42$ the variation of $\mathrm{V}$ group elements content $\mathrm{y}$ with the epilayer depth were revealed, weher the compositions of V-group elements were changed up to $\Delta y=0.1$ atomic fractions in $\mathrm{V}$ group elements sublattice. In most cases, $y$ change occurs in a GaInAsP region up to $200 \mathrm{~nm}$ thick adjacent to the InP. In some cases, y changes throughout the whole GaInPAs layer thickness. Fo the epitaxial layers with a satisfactory crystal perfection the less was the mismatch between the substrate and the GaInPAs epitaxial layer, the smaller was the value of $\Delta y$. For GaInPAs layers characterized by a low degree of crystal perfection and a high lattice mismatch between GaInAsP and InP layers, the value of $\Delta y$ was about zero. These data let us suggest that the incorporation of atoms of the V group in the epitaxial layer strongly depends on elastic deformation of the growing monolayer, that is mismatched with the underlying crystal surface. 